\title{
Searching for New Physics in Two-Neutrino Double Beta Decay
}

\author{
Frank F. Deppisch $\oplus^{1, *}$ Lukas Graf $\odot,{ }^{2, \dagger}$ and Fedor Šmkovic $\oplus^{3,4,5, *}$ \\ ${ }^{1}$ Department of Physics and Astronomy, University College London, London WC1E 6BT, United Kingdom \\ ${ }^{2}$ Max-Planck-Institut für Kernphysik, Saupfercheckweg 1, 69117 Heidelberg, Germany \\ ${ }^{3}$ BLTP, JINR, 141980 Dubna, Russia \\ ${ }^{4}$ Comenius University, Mlynská dolina F1, SK842 48 Bratislava, Slovakia \\ ${ }^{5}$ IEAP CTU, 12800 Prague, Czech Republic
}

(Received 2 April 2020; accepted 16 September 2020; published 19 October 2020)

\begin{abstract}
Motivated by nonzero neutrino masses and the possibility of new physics discovery, a number of experiments search for neutrinoless double beta decay. While hunting for this hypothetical nuclear process, a significant amount of two-neutrino double beta decay data have become available. Although these events are regarded and studied mostly as the background of neutrinoless double beta decay, they can also be used to probe physics beyond the standard model. In this Letter, we show how the presence of right-handed leptonic currents would affect the energy distribution and angular correlation of the outgoing electrons in two-neutrino double beta decay. Consequently, we estimate constraints imposed by currently available data on the existence of right-handed neutrino interactions without having to assume their nature. In this way, our results complement the bounds coming from the nonobservation of neutrinoless double beta decay as they limit also the exotic interactions of Dirac neutrinos. We perform a detailed calculation of two-neutrino double beta decay under the presence of exotic (axial-) vector currents, and we demonstrate that current experimental searches can be competitive to existing limits.
\end{abstract}

DOI: 10.1103/PhysRevLett.125.171801

Introduction.-Double beta decay processes are sensitive probes of physics beyond the standard model (SM). The SM process of two-neutrino double beta $(2 \nu \beta \beta)$ decay is among the rarest processes ever observed with half-lives of order $T_{1 / 2}^{2 \nu \beta \beta} \sim 10^{19} \mathrm{yr}$ and longer [1]. Neutrinoless double beta $(0 \nu \beta \beta)$ decay, with no observation of any missing energy, is clearly the most important process beyond the SM, as it probes the Majorana nature and mass $m_{\nu}$ of light neutrinos, with current experiments sensitive as $T_{1 / 2}^{0 \nu \beta \beta} \sim\left(0.1 \mathrm{eV} / m_{\nu}\right)^{2} \times 10^{26} \mathrm{yr}$. In general, it is a crucial test for any new physics scenario that violates lepton number by two units [2-4].

While $0 \nu \beta \beta$ decay is the key process, experimental searches for this decay also provide a detailed measurement of the $2 \nu \beta \beta$ decay rate and spectrum in several isotopes. For example, KamLAND-Zen measures the $2 \nu \beta \beta$ decay spectrum in ${ }^{136} \mathrm{Xe}$ with a high statistics [5] but can only do it with respect to the sum of energies of the two electrons emitted. On the other hand, the NEMO-3 experiment with the technology to track individual electrons can measure the individual electron energy spectra and the opening

Published by the American Physical Society under the terms of the Creative Commons Attribution 4.0 International license. Further distribution of this work must maintain attribution to the author(s) and the published article's title, journal citation, and DOI. Funded by SCOAP ${ }^{3}$. angle between the two electrons. This has yielded detailed measurements of the $2 \nu \beta \beta$ decay spectra of ${ }^{96} \mathrm{Zr}[6],{ }^{150} \mathrm{Nd}$ [7], ${ }^{48} \mathrm{Ca}[8],{ }^{82} \mathrm{Se}$ [9], and especially ${ }^{100} \mathrm{Mo} \mathrm{[10],} \mathrm{the} \mathrm{latter}$ with a very high statistics containing $\approx 5 \times 10^{5} 2 \nu \beta \beta$ decay events. Such measurements are important for the interpretation of $0 \nu \beta \beta$ decay searches, as it can shed light on the value of the effective axial coupling $g_{A}$ [11].

The high precision of $2 \nu \beta \beta$ decay measurements expected to continue as the experimental exposures are increased to push the sensitivity of $0 \nu \beta \beta$ decay searches begs the question whether $2 \nu \beta \beta$ decay events can be directly used to search for new physics beyond the SM. This is the focus of this work. We model such new physics effects through effective charged-current operators of the form $\epsilon G_{F}\left(\bar{e} \mathcal{O}_{1} \nu\right)\left(\bar{u} \mathcal{O}_{2} d\right)$ with Lorentz structures $\mathcal{O}_{1}, \mathcal{O}_{2}$ other than the SM $V-A$ type. Here, the Fermi constant $G_{F}$ is introduced, and the small dimensionless coupling $\epsilon$ encapsulates the new physics effects.

Exotic charged-current operators of the above form are being searched for in nuclear, neutron $\beta$, and pion decays as well as collider searches [12], giving rise to limits of the order $\epsilon \lesssim 10^{-4}-10^{-1}$, depending on the Lorentz structure and chirality of the fields involved. In this Letter, we will specifically concentrate on exotic operators containing right-handed $(\mathrm{RH})$ vector lepton currents. Such operators prove difficult to constrain as interference with the SM contribution is suppressed by the light neutrino masses. They are nevertheless of strong theoretical interest as their 
observation, along with the nonobservation of lepton number violation, would indicate that neutrinos are not Majorana fermions. This is because RH currents with neutrinos but in the absence of a sterile neutrino state would necessarily violate lepton number. In this Letter, we will show that the existing $2 \nu \beta \beta$ data from the NEMO-3 experiment may set the most stringent limits on such operators which are currently only weakly constrained at the $6 \%$ level [12]. We thus describe a novel probe of the fundamental nature of weak interactions and the properties of neutrinos.

Exotic charged-current interactions.-We are interested in processes where right- and left-handed electrons are emitted considering only $(V+A)$ and $(V-A)$ currents. The effective Lagrangian is written as

$$
\begin{aligned}
\mathcal{L}= & \frac{G_{F} \cos \theta_{C}}{\sqrt{2}}\left[\left(1+\delta_{\mathrm{SM}}+\epsilon_{\mathrm{LL}}\right) j_{L}^{\mu} J_{L \mu}+\epsilon_{\mathrm{RL}} j_{L}^{\mu} J_{R \mu}\right. \\
& \left.+\epsilon_{\mathrm{LR}} j_{R}^{\mu} J_{L \mu}+\epsilon_{\mathrm{RR}} j_{R}^{\mu} J_{R \mu}\right]+ \text { H.c. },
\end{aligned}
$$

with the tree-level Fermi constant $G_{F}$, the Cabbibo angle $\theta_{C}$, and the leptonic and hadronic currents $j_{L, R}^{\mu}=$ $\bar{e} \gamma^{\mu}\left(1 \mp \gamma_{5}\right) \nu$ and $J_{L, R}^{\mu}=\bar{u} \gamma^{\mu}\left(1 \mp \gamma_{5}\right) d$, respectively. The $\mathrm{SM}$ electroweak radiative corrections are encoded in $\delta_{\mathrm{SM}}$, and the $\epsilon_{X Y}$ encapsulate new physics effects. Here we concentrate on the latter two operators with $\mathrm{RH}$ lepton currents, as they are expected to change the $2 \nu \beta \beta$ decay kinematic spectra more significantly. Extensions of the above set of operators can be considered; for example, currents other than vector and axial-vector can be included [13] and further exotic particles may participate [14].

In Eq. (1), $\nu$ is a four-spinor field of the light electron neutrino, either defined by $\nu=\nu_{L}+\nu_{L}^{c}$ (i.e., a Majorana spinor constructed from the SM active left-handed neutrino $\nu_{L}$ and its charge conjugate) or $\nu=\nu_{L}+\nu_{R}$ (a Dirac spinor constructed from the SM $\nu_{L}$ and a new SM-sterile RH neutrino $\nu_{R}$ ). Whether the light neutrinos are of Majorana or Dirac type and whether total lepton number is broken or conserved is of crucial importance for an underlying model, but as far as the effective interactions in Eq. (1) are concerned, this does not play a role in our calculations. If the neutrino in Eq. (1) is a Majorana particle, the operators associated with $\epsilon_{\mathrm{LR}}$ and $\epsilon_{\mathrm{RR}}$ violate total lepton number by two units, and they will give rise to extra contributions to $0 \nu \beta \beta$ decay [15]. In this case, severe limits are set by $0 \nu \beta \beta$ decay searches of the order $\epsilon_{\mathrm{LR}} \lesssim 3 \times 10^{-9}$, $\epsilon_{\mathrm{RR}} \lesssim 6 \times 10^{-7}$ [2]. On the other hand, if there exists a sterile neutrino Weyl state $\nu_{R}$ that combines with $\nu_{L}$ to form a Dirac neutrino, the RH current interactions in Eq. (1) do not necessarily violate lepton number which, in fact, can remain an unbroken symmetry of the underlying model. For example, such effective interactions can emerge in leftright symmetric models (LRSMs) [16] with unbroken lepton number [17]. The observation of the effect of RH neutrino operators without the observation of lepton number violation would thus strongly suggest that neutrinos are Dirac fermions.

The most stringent direct limits on the above operators for process energies $E \approx \mathrm{MeV}$ are set by fitting experimental results of neutron and various nuclear single $\beta$ decays, $\epsilon_{\mathrm{LL}}, \epsilon_{\mathrm{RL}} \leq 5 \times 10^{-4}, \epsilon_{\mathrm{LR}}, \epsilon_{\mathrm{RR}} \leq 6 \times 10^{-2}[12,13]$. The limits on the RH lepton currents are much less severe due to the absence of an interference with the SM contribution. Searches at the Large Hadron Collider (LHC) for single electron and missing energy signatures [18] $p p \rightarrow e X+$ MET may also be used to constrain the above operators, $\epsilon_{\mathrm{LL}} \lesssim 4.5 \times 10^{-3}, \epsilon_{\mathrm{RR}} \lesssim 2.2 \times 10^{-3}$ [19]. While the constraints are stringent, and the sensitivity is expected to improve to $\epsilon_{\mathrm{LL}} \approx 10^{-5}$ [20], the LHC operates at a much higher energy, and the effective operator analysis is only applicable if the new physics mediators integrated out are much heavier than this. More model-dependent limits can also be set by direct searches for RH current mediators at the LHC [21], from considerations of sterile neutrino thermalization and the resulting increase of the effective number of light degrees of freedom in the early Universe and supernova cooling. The associated new physics scales probed range between $\Lambda \approx 5$ and $20 \mathrm{TeV}$, corresponding to $\epsilon_{X Y} \approx\left(5 \times 10^{-4}\right)-\left(5 \times 10^{-5}\right)$. An indirect limit on $\epsilon_{\mathrm{LR}}$ can be set from the fact that the associated operator contributes to the Dirac neutrino mass at the second loop order [22]. Using current direct neutrino mass bounds, this results in $\epsilon_{\mathrm{LR}} \lesssim 10^{-2}$ [23]. In particular, the direct limit $\epsilon_{\mathrm{LR}} \leq 6 \times 10^{-2}$ is rather feeble and motivates the need to probe for admixtures of exotic currents in the SM Fermi interaction. While underlying scenarios are expected to trigger the other better-constrained operators as well, it is not difficult to envision cases where $\epsilon_{\mathrm{LR}}$ or $\epsilon_{\mathrm{RR}}$ are dominant. For example, in LRSMs, the operator associated with $\epsilon_{\mathrm{RL}}$ is mediated at lowest order by the SM $W$ boson and involves the mixing $\theta_{\mathrm{LR}}$ with an exotic $W_{R}$ boson. This mixing is a priori unrelated to the $m_{W_{R}}$ scale, and $\epsilon_{\mathrm{RL}}$ can thus be suppressed compared to $\epsilon_{\mathrm{RR}}$ if $\theta_{\mathrm{LR}}$ is small. It is also not difficult to think of extensions of the minimal LRSM where exotic copies of quarks are charged under the LRSM SU $(2)_{R}$ but not the SM quarks. The exotic quarks instead mix with the SM quarks, and the latter will inherit a suppressed RH current, suppressing $\epsilon_{\mathrm{RL}}$ with respect to $\epsilon_{\mathrm{LR}}$.

Decay rate and distributions. - We have calculated the differential rate of $2 \nu \beta \beta$ decay under the presence of the exotic interactions in Eq. (1). Because $2 \nu \beta \beta$ decay is possible in the SM, arising in second order perturbation theory of the first term in Eq. (1), interference between SM and exotic contributions is in principle possible. In general, the amplitude of $2 \nu \beta \beta$ decay is calculated as a coherent sum of the Feynman diagrams in Fig. 1. To lowest order in $\epsilon_{X R}$, exotic effects occur from the interference of the SM diagram Fig. 1 (left) and Fig. 1 (center). Because of the 

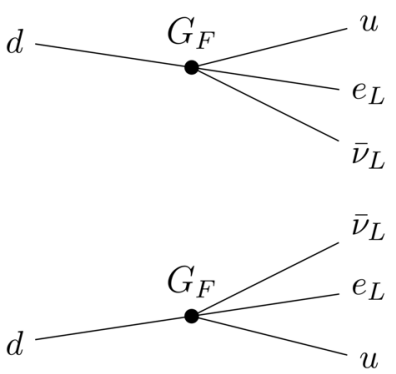
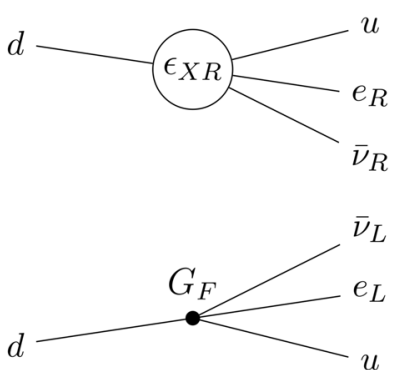
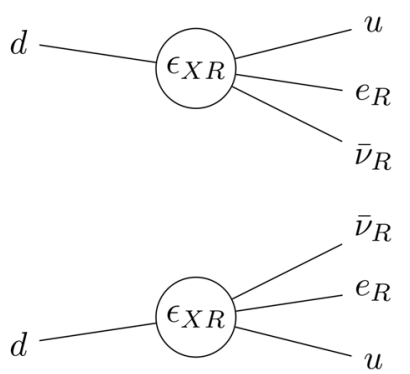

FIG. 1. Feynman diagrams for ordinary $2 \nu \beta \beta$ decay via the second-order transition through the SM $V-A$ interaction with strength given by the Fermi constant $G_{F}$ (left), a transition involving one exotic interaction $\epsilon_{X R} G_{F}$ with a $V+A$ lepton current of the form $\left(\bar{e}_{R} \mathcal{O}_{1} \nu\right)\left(\bar{u} \mathcal{O}_{2} d\right)$ (center), and a second-order transition through the same exotic interaction (right).

RH nature of the exotic current, such an interference is helicity suppressed by the masses of the emitted electron and neutrino as $m_{e} m_{\nu} / Q^{2}$, with the $2 \nu \beta \beta$ decay energy release $Q$. For light $\mathrm{eV}$-scale neutrinos, it is thus utterly negligible. [This is not necessarily the case if currents other than $V \pm A$ vector currents are considered in Eq. (1).] Contributions to second order $\propto \epsilon_{X R}^{2}$ come from the center diagram and the interference of the SM contribution (left) with the second-order exotic diagram (right). The latter is suppressed even more strongly by the neutrino mass and thus negligible. To lowest order in the exotic coupling, the squared matrix element for ground state to ground state $2 \nu \beta \beta$ transition can thus be written as the incoherent sum

$$
\left|\mathcal{R}^{2 \nu}\right|^{2}=\left|\mathcal{R}_{\mathrm{SM}}^{2 \nu}\right|^{2}+\left|\epsilon_{X R}\right|^{2}\left|\mathcal{R}_{\epsilon}^{2 \nu}\right|^{2},
$$

where $\mathcal{R}_{\mathrm{SM}}^{2 \nu}$ is the matrix element for SM $2 \nu \beta \beta$ decay and $\mathcal{R}_{\epsilon}^{2 \nu}$ is the exotic contribution. As discussed in detail in the Supplemental Material [24], the latter may be expressed as

$$
\begin{aligned}
\mathcal{R}_{\epsilon}^{2 \nu}= & i\left(\frac{1}{\sqrt{2}}\right)^{2}\left(\frac{G_{F} \cos \theta_{W}}{\sqrt{2}}\right)^{2}\left[1-P\left(e_{1}, e_{2}\right)\right]\left[1-P\left(\bar{\nu}_{1}, \bar{\nu}_{2}\right)\right] \\
& \times\left[\bar{\psi}\left(p_{e_{1}}\right) \gamma^{\mu}\left(1+\gamma_{5}\right) \psi^{c}\left(p_{\bar{\nu}_{1}}\right) \bar{\psi}\left(p_{e_{2}}\right) \gamma^{\nu}\left(1-\gamma_{5}\right) \psi^{c}\left(p_{\bar{\nu}_{2}}\right)\right. \\
& \left.+\bar{\psi}\left(p_{e_{1}}\right) \gamma^{\nu}\left(1-\gamma_{5}\right) \psi^{c}\left(p_{\bar{\nu}_{1}}\right) \bar{\psi}\left(p_{e_{2}}\right) \gamma^{\mu}\left(1+\gamma_{5}\right) \psi^{c}\left(p_{\bar{\nu}_{2}}\right)\right] \\
& \times\left(g_{\mu 0} g_{\nu 0} g_{V}^{2} M_{F} \mp \frac{1}{3} g_{\mu k} g_{\nu k} g_{A}^{2} M_{\mathrm{GT}}\right)
\end{aligned}
$$

where $\psi\left(p_{f}\right)$ is the wave function of the emitted fermion $f$ with momentum $p_{f}$, and we consider here the commonly used approximation of the $S_{1 / 2}$ wave evaluated at the nuclear surface. The nuclear matrix elements between the initial $0_{i}^{+}$, the intermediate $0_{n}^{+}\left(1_{n}^{+}\right)$, and the final $0_{f}^{+}$states of the nucleus are generally of Fermi (Gamow-Teller) type with the associated nucleon-level vector (effective axialvector) coupling $g_{V}\left(g_{A}\right)$,

$$
\begin{aligned}
M_{F} & =\sum_{n} \frac{\left\langle 0_{f}^{+}\left|\sum_{j} \tau_{j}^{+}\right| 0_{n}^{+}\right\rangle\left\langle 0_{n}^{+}\left|\sum_{k} \tau_{k}^{+}\right| 0_{i}^{+}\right\rangle}{\Delta E_{n}\left(0_{n}^{+}\right)+E_{e_{2}}+E_{\bar{\nu}_{2}}}, \\
M_{\mathrm{GT}} & =\sum_{n} \frac{\left\langle 0_{f}^{+}\left|\sum_{j} \tau_{j}^{+} \boldsymbol{\sigma}_{j}\right| 1_{n}^{+}\right\rangle\left\langle 1_{n}^{+}\left|\sum_{k} \tau_{k}^{+} \boldsymbol{\sigma}_{k}\right| 0_{i}^{+}\right\rangle}{\Delta E_{n}\left(1_{n}^{+}\right)+E_{e_{2}}+E_{\bar{\nu}_{2}}} .
\end{aligned}
$$

The summations are over all intermediate $0_{n}^{+}, 1_{n}^{+}$states and all nucleons $j, k$ inside the nucleus where $\tau_{j, k}^{+}$is the isospinraising operator transforming a neutron into a proton and $\boldsymbol{\sigma}_{j, k}$ represents the nucleon spin operator. Assuming isospin invariance, the Fermi matrix elements vanish. The energy denominators arise due to the second-order nature of the above matrix element where $\Delta E_{n}\left(J_{n}^{\pi}\right)=E_{n}\left(J_{n}^{\pi}\right)-E_{i}$ $\left(J_{n}^{\pi}=0_{n}^{+}\right.$and $\left.1_{n}^{+}\right)$are the energies of the intermediate nuclear states with respect to the initial ground state. Overall energy conservation is implied $E_{i}=E_{f}+E_{e_{1}}+E_{e_{2}}+E_{\bar{\nu}_{1}}+E_{\bar{\nu}_{2}}$, and, as indicated by the particle exchange operator $P(a, b)$, the matrix element is antisymmetrized with respect to the exchange of the identical electrons and antineutrinos (the corresponding antisymmetrization over the nucleons is implicitly included in the nuclear states).

Following Ref. [11], the calculation of the $2 \nu \beta \beta$ decay rate and distributions is detailed in the Supplemental Material [24]. We use nuclear matrix elements in the quasiparticle random phase approximation (QRPA) formalism from Ref. [11] assuming isospin invariance with $M_{F}=0$ and including higher-order corrections from the effect of the final state lepton energies. Because of $M_{F}=0$ and negligible effects of interference between the SM and exotic amplitudes, the calculations for $\epsilon_{\mathrm{LR}}$ and $\epsilon_{\mathrm{RR}}$ are identical; both cases yield the same rates and distributions. As a result, we calculate the full differential $2 \nu \beta \beta$ decay rate in a given $0^{+} \rightarrow 0^{+}$double beta decaying isotope with respect to the two electron energies $m_{e} \leq E_{e_{1}, e_{2}} \leq Q+m_{e}$ and the angle $0 \leq \theta \leq \pi$ between the emitted electrons, which may be written as

$\frac{d \Gamma^{2 \nu}}{d E_{e_{1}} d E_{e_{2}} d \cos \theta}=\frac{\Gamma^{2 \nu}}{2} \frac{d \Gamma_{\text {norm }}^{2 \nu}}{d E_{e_{1}} d E_{e_{2}}}\left[1+\kappa^{2 \nu}\left(E_{e_{1}}, E_{e_{2}}\right) \cos \theta\right]$. 

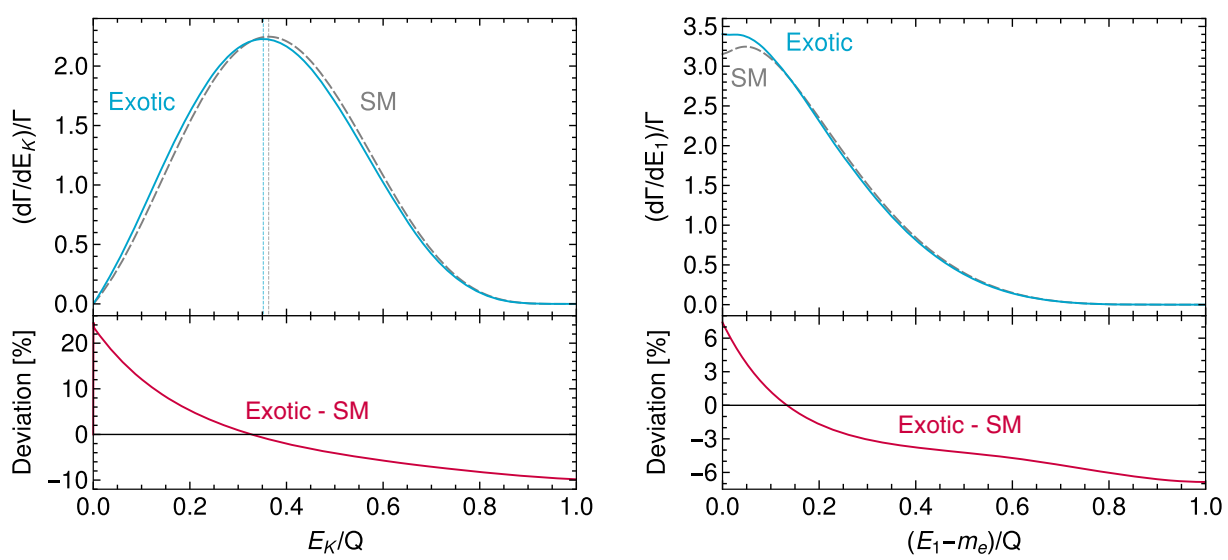

FIG. 2. Left: normalized $2 \nu \beta \beta$ decay distributions with respect to the total kinetic energy $E_{K}=E_{e_{1}}+E_{e_{2}}-2 m_{e}$ of the emitted electrons for standard $2 \nu \beta \beta$ decay through SM $V-A$ currents (dashed) and a pure RH lepton current (solid). Right: normalized $2 \nu \beta \beta$ decay distributions with respect to the energy of a single electron in the same scenarios. Both plots are for the isotope ${ }^{100} \mathrm{Mo}$ and the energies are normalized to the $Q$ value. The bottom panels show the relative deviation of the exotic distribution from the SM case.

Because interference effects between the SM and the $\mathrm{RH}$ current diagram are negligible, the differential rate is simply the incoherent sum of both. In the Supplemental Material [24], we describe in detail the calculation of the above differential decay rate and the derived energy distributions, angular correlations, and total rate. Specifically, for ${ }^{100}$ Mo the total decay rate $\Gamma^{2 \nu}=\ln 2 / T_{1 / 2}^{2 \nu}$ associated with the $2 \nu \beta \beta$ half-life $T_{1 / 2}^{2 \nu}$ may be approximated as $\Gamma^{2 \nu} \approx \Gamma_{\mathrm{SM}}^{2 \nu}\left(1+6.11 \epsilon_{X R}^{2}\right)$, where $\Gamma_{\mathrm{SM}}^{2 \nu}$ is the SM rate. The experimentally accessible kinematic information is contained in the normalized double-differential energy distribution $d \Gamma_{\text {norm }}^{2 \nu} /\left(d E_{e_{1}} d E_{e_{2}}\right)$ and the energy-dependent angular correlation $-1<\kappa^{2 \nu}\left(E_{e_{1}}, E_{e_{2}}\right)<1$. The latter determines whether the two electrons are preferably emitted back to back $\left(\kappa^{2 \nu} \approx-1\right)$, in the same direction $\left(\kappa^{2 \nu} \approx 1\right)$, or in intermediate configurations.

Given the uncertainties in nuclear matrix elements, the change of the total decay rate due to the presence of a $\mathrm{RH}$ current contribution is not expected to be measurable. Instead, differences in spectral shape of either the energy or angular distributions may be more sensitive. All double beta decay experiments measure the spectrum of events with respect to the sum of the electron kinetic energies, $E_{K}=E_{e_{1}}+E_{e_{2}}-2 m_{e}$. For ${ }^{100} \mathrm{Mo}$, it is shown in Fig. 2 (left) comparing the $2 \nu \beta \beta$ decay distributions in the SM case (dashed) and for the exotic leptonic RH current operators (solid). The deviation is sizeable leading to a shift of the spectrum to smaller energies and a flatter profile near the end point $E_{K} / Q=1$. We find that relative deviations of the order of $10 \%$ for small energies and near the end point are expected to occur. In experiments that are able to track and measure the individual electrons, such as NEMO-3 and SuperNEMO, the full doubly differential energy spectrum is in principle measurable. Alternatively, the spectrum with respect to the kinetic energy of a single electron is shown in Fig. 2 (right). It helps explain the shift of the energy sum spectrum in the exotic case, as each electron receives on average less energy than in the SM.

This behavior can be traced to the kinematic differences. In the presence of a $\mathrm{RH}$ lepton current in $2 \nu \beta \beta$ decay, the electrons are preferably emitted collinearly and the electron energy-dependent correlation factor is always $\kappa_{\epsilon}^{2 \nu}>0$, whereas in the SM case the electrons are preferably emitted back to back with $\kappa_{\mathrm{SM}}^{2 \nu}<0$. This behavior can be understood from angular momentum considerations when the two electrons are produced with opposite dominant helicities. Integrating Eq. (5) over the electron energies, one arrives at the angular distribution,

$$
\frac{d \Gamma^{2 \nu}}{d \cos \theta}=\frac{\Gamma^{2 \nu}}{2}\left(1+K^{2 \nu} \cos \theta\right),
$$

with the angular correlation factor $K^{2 \nu}$. For ${ }^{100} \mathrm{Mo}$, we calculate $K_{\mathrm{SM}}^{2 \nu}=-0.626$ in the SM and $K_{\epsilon}^{2 \nu}=+0.370$ for the exotic contribution. This deviation is clearly the most striking consequence of a RH lepton current on $2 \nu \beta \beta$ decay. For small $\epsilon_{X R} \ll 1$, the angular correlation factor $K^{2 \nu}$ can be expanded as

$$
K^{2 \nu}=K_{\mathrm{SM}}^{2 \nu}+\alpha \epsilon_{X R}^{2}+\mathcal{O}\left(\epsilon_{X R}^{4}\right) .
$$

For ${ }^{100} \mathrm{Mo}$, the coefficient $\alpha$ turns out to be $\alpha=6.078$. Despite the small correction expected, if $\epsilon_{X R} \approx 10^{-2}$ as indicated in current bounds, searches for $2 \nu \beta \beta$ decay can be sensitive in this regime. A simple signature is to look for the forward-backward asymmetry $A_{\theta}^{2 \nu}$, comparing the number of $2 \nu \beta \beta$ decay events with the electrons being emitted with a relative angle $\theta<\pi / 2$ and $\theta>\pi / 2$,

$$
A_{\theta}^{2 \nu}=\frac{N_{\theta>\pi / 2}-N_{\theta<\pi / 2}}{N_{\theta>\pi / 2}+N_{\theta<\pi / 2}}=\frac{1}{2} K^{2 \nu} .
$$


As shown, the asymmetry is simply related to the angular correlation factor $K^{2 \nu}$, and it is clearly independent of the overall $2 \nu \beta \beta$ decay rate. Considering only the statistical error, with $N_{\text {events }}=5 \times 10^{5} 2 \nu \beta \beta$ decay events at NEMO3 , the angular correlation coefficient should be measurable with an uncertainty $K_{\mathrm{SM}}^{2 \nu}=-0.6260 \pm 0.0027$. No significant deviation from this SM expectation should then constrain $\epsilon_{X R} \lesssim 2.7 \times 10^{-2}$ at $90 \%$ confidence level. This would already improve on the single $\beta$ decay constraint of $6 \times 10^{-2}$ [13]. If an experiment such as SuperNEMO were able to achieve an increase in exposure by 3 orders of magnitude, the expected future sensitivity scaling as $1 / \sqrt{N_{\text {events }}}$ would be $\epsilon_{X R} \lesssim 4.8 \times 10^{-3}$. This only gives a very rough order of magnitude estimate, and a dedicated experimental analysis is required to verify the sensitivity. For example, at NEMO-3 and SuperNEMO, detector effects will result in a reduced acceptance for small electron angles, thus affecting the systematic uncertainty $[10,28]$. We note though that it is not strictly necessary to measure the forward-backward asymmetry in Eq. (8). Instead, even if only including events with $\cos \theta<0$, where the majority of $2 \nu \beta \beta$ events occur, will allow us to fit the angular distribution in Eq. (6), albeit with a lower statistical significance. In this back-to-back region with $\cos \theta \lesssim 0$, the existing NEMO-3 data are well within the statistical fluctuations [10]. As a very rough but conservative estimate, dropping half of the events will give a dataset limited by statistics. This would weaken our estimated sensitivity by a factor of $\sqrt{2}$.

We must also consider the theoretical uncertainty in predicting the angular correlation. Our results were calculated within the nuclear structure framework of the protonneutron QRPA with partial isospin restoration [11]. We consider the three main sources of theoretical errors: (i) The spectrum of intermediate nuclear states as calculated in different nuclear structure models has a small but potentially significant impact on the external lepton phase space and thus the angular correlation. To conservatively model this, we vary the effective axial coupling $g_{A}$ between $g_{A}=$ 0.8 and $g_{A}=1.269$ as described in Ref. [11]. This drastically changes the associated ${ }^{100}$ Mo matrix element by a factor of 2.6 and thus the decay rate by a factor of $\approx 6.8$, but the SM angular correlation changes only as $K_{\mathrm{SM}}^{2 \nu}=-0.6260 \pm 0.0030$. Thus, the very conservatively estimated theoretical error is of the same order as the current statistical error. It will be crucial to reduce it to match the improved future statistical uncertainty, though. (ii) In Eq. (1), we only include the fundamental parton-level interactions, and we neglect higher-order nuclear currents, namely, the induced weak magnetism and pseudoscalar currents. Their dominant effect on the amplitude will occur in the interference between the latter and the axial-vector nuclear current, which is suppressed by $\lesssim Q^{2} /\left(3 m_{\pi}^{2}\right) \approx 2 \times$ $10^{-4}$ [29], where $m_{\pi}$ is the pion mass. This results in a currently negligible correction. (iii) For simplicity, we analytically treat the outgoing electron wave functions in the so-called Fermi approximation. The proper Coulomb interaction with the nucleus and the electron cloud can be calculated numerically [30], leading to a $15 \%$ correction in the resulting phase space factor but only a negligible shift in the SM angular correlation of $0<\Delta K_{\mathrm{SM}}^{2 \nu} \lesssim 10^{-4}[30]$.

As can be seen in Fig. 2 (right), the effect of RH currents is similar to that of varying the contribution of intermediate nuclear states as described in Ref. [11]. It exhibits a similar variation for small electron energies near the peak, depending on single state dominance vs higher state dominance modeling of the intermediate nuclear state contributions [10]. This has the benefit that experimental searches for these effects, such as described in Refs. [5,10,31], could be adapted to our scenario.

Conclusions.-Nuclear double beta decay with the emission of two neutrinos and nothing else was proposed over 80 years ago [32] as a consequence of the Fermi theory of single $\beta$ decay. Its main role for particle physics has largely been confined to being an irreducible background to the exotic and yet unobserved lepton number violating neutrinoless $(0 \nu \beta \beta)$ mode. We have demonstrated here, for the first time to our knowledge, that $2 \nu \beta \beta$ decay may be used in its own right as a probe of new physics. Our result shows that searches for deviations in the spectrum of $2 \nu \beta \beta$ decay can be competitive to existing limits. This provides a motivation to utilize the already large set of observed $2 \nu \beta \beta$ decay events to probe exotic scenarios. The number of events will necessarily increase in the future by 1 to 2 orders of magnitude, as $0 \nu \beta \beta$ decay is being searched for in future experiments.

Here we have focused on the case of effective operators with RH chiral neutrinos where the interference with the SM contributions is negligible due to the suppression by the neutrino mass. The exotic contribution to observables is therefore proportional to the square of the small new physics parameter. As a result, such operators are comparatively weakly constrained. They still play an important role in our understanding of neutrinos as the RH nature can be accommodated in one of two ways: (i) through the rightchiral part of the SM neutrino as a Majorana fermion, in which case, the associated operators will also induce the lepton number violating $0 \nu \beta \beta$ decay mode at a level that is already ruled out or (ii) through the presence of a separate RH neutrino state that, while sterile under the SM gauge interactions, participates in exotic interactions beyond the SM. In the latter case, neutrinos are expected to be Dirac fermions and the observation of $\mathrm{RH}$ neutrino currents, while the lepton number violating $0 \nu \beta \beta$ decay is not observed would indicate this scenario.

If other operators such as scalar currents are considered, interference can be sizeable and even larger effects may be seen, although existing limits such as those from single $\beta$ decay are expected to be more restrictive as well. As we 
have demonstrated in the example of exotic $\mathrm{RH}$ vector currents, while the search for $0 \nu \beta \beta$ decay and thus the Majorana nature of neutrinos is the main motivation, the properties of the second-order SM process of $2 \nu \beta \beta$ decay can also contain potential hints for new physics.

F. F. D. acknowledges support from the UK Science and Technology Facilities Council via a Consolidated Grant (Reference No. ST/P00072X/1). F. F. D. is grateful to the Institut für Hochenergiephysik (HEPHY) Vienna and together with L. G. to the Comenius University Bratislava where part of the work was completed. F. F. D. would also like to thank Morten Sode for collaboration in the early stages of the project. F. $\breve{S}$. acknowledges support by the Vedecká grantová agentúra (VEGA) Grant Agency of the Slovak Republic under Contract No. 1/0607/20 and by the Ministry of Education, Youth and Sports of the Czech Republic under the Inženýrské aplikace fyziky mikrosvěta (INAFYM) Grant No. CZ.02.1.01/0.0/0.0/ 16_019/0000766.

*f.deppisch@ucl.ac.uk

†lukas.graf@mpi-hd.mpg.de

\#fedor.simkovic@fmph.uniba.sk

[1] A. Barabash, AIP Conf. Proc. 2165, 020002 (2019).

[2] F. F. Deppisch, M. Hirsch, and H. Päs, J. Phys. G 39, 124007 (2012)

[3] L. Graf, F. F. Deppisch, F. Iachello, and J. Kotila, Phys. Rev. D 98, 095023 (2018).

[4] V. Cirigliano, W. Dekens, J. de Vries, M. L. Graesser, and E. Mereghetti, J. High Energy Phys. 12 (2018) 097.

[5] A. Gando et al. (KamLAND-Zen Collaboration), Phys. Rev. Lett. 122, 192501 (2019).

[6] J. Argyriades et al. (NEMO-3 Collaboration), Nucl. Phys. A847, 168 (2010).

[7] R. Arnold et al. (NEMO-3 Collaboration), Phys. Rev. D 94, 072003 (2016).

[8] R. Arnold et al. (NEMO-3 Collaboration), Phys. Rev. D 93, 112008 (2016).

[9] R. Arnold et al., Eur. Phys. J. C 78, 821 (2018).
[10] R. Arnold et al. (NEMO-3 Collaboration), Eur. Phys. J. C 79, 440 (2019).

[11] F. Šimkovic, R. Dvornický, D. Štefánik, and A. Faessler, Phys. Rev. C 97, 034315 (2018).

[12] M. Gonzalez-Alonso, O. Naviliat-Cuncic, and N. Severijns, Prog. Part. Nucl. Phys. 104, 165 (2019).

[13] V. Cirigliano, S. Gardner, and B. Holstein, Prog. Part. Nucl. Phys. 71, 93 (2013).

[14] R. Cepedello, F. F. Deppisch, L. Gonzalez, C. Hati, and M. Hirsch, Phys. Rev. Lett. 122, 181801 (2019).

[15] M. Doi, T. Kotani, H. Nishiura, and E. Takasugi, Prog. Theor. Phys. 69, 602 (1983).

[16] J. C. Pati and A. Salam, Phys. Rev. D 10, 275 (1974).

[17] P. D. Bolton, F. F. Deppisch, C. Hati, S. Patra, and U. Sarkar, Phys. Rev. D 100, 035013 (2019).

[18] V. Khachatryan et al. (CMS Collaboration), Phys. Rev. D 91, 092005 (2015).

[19] O. Naviliat-Cuncic and M. Gonzalez-Alonso, Ann. Phys. (Amsterdam) 525, 600 (2013).

[20] A. Greljo and D. Marzocca, Eur. Phys. J. C 77, 548 (2017).

[21] M. Aaboud et al. (ATLAS Collaboration), J. High Energy Phys. 01 (2019) 016.

[22] G. Prezeau and A. Kurylov, Phys. Rev. Lett. 95, 101802 (2005).

[23] K. K. Vos, H. W. Wilschut, and R. G. E. Timmermans, Rev. Mod. Phys. 87, 1483 (2015).

[24] See Supplemental Material at http://link.aps.org/ supplemental/10.1103/PhysRevLett.125.171801 for a detailed calculation of the $2 \nu \beta \beta$ decay rate under the presence of exotic right-handed currents, which include Refs. [25-27].

[25] M. Doi, T. Kotani, and E. Takasugi, Prog. Theor. Phys. Suppl. 83, 1 (1985).

[26] W. Haxton and G. Stephenson, Prog. Part. Nucl. Phys. 12, 409 (1984).

[27] P. Gysbers et al., Nat. Phys. 15, 428 (2019).

[28] R. Arnold et al. (SuperNEMO Collaboration), Eur. Phys. J. C 70, 927 (2010).

[29] T. Tomoda, Rep. Prog. Phys. 54, 53 (1991).

[30] J. Kotila and F. Iachello, Phys. Rev. C 85, 034316 (2012).

[31] O. Azzolini, J. W. Beeman, F. Bellini, M. Beretta, M. Biassoni et al., Phys. Rev. Lett. 123, 262501 (2019).

[32] M. Goeppert-Mayer, Phys. Rev. 48, 512 (1935). 